\title{
Misunderstanding of Pre-Exposure Prophylaxis Use Among Men Who Have Sex with Men: Public Health and Policy Implications
}

\author{
Steven P. Kurtz, PhD and Mance E. Buttram, PhD
}

\begin{abstract}
Purpose: Street markets in antiretroviral medications for HIV have been documented, but sources of demand are not well understood. We report unexpected findings from qualitative research suggesting that some demand is for informal pre-exposure prophylaxis (PrEP).

Methods: Focus groups with young men who have sex with men $(N=31)$ yielded information on their understanding and use of PrEP.

Results: Of those who had heard of it, few understood PrEP to be a physician-prescribed regimen; most believed it to be a pill taken before and/or after sex and acquired on the street or through HIV-positive friends.
\end{abstract}

Conclusion: Implications for PrEP rollout and public health policy are discussed.

Keywords: drug diversion, HIV prevention, MSM, PrEP

\section{Introduction}

D IVERSION - THE UNLAWFUL CHANNELING of regulated pharmaceuticals to illicit markets 1 - of antiretrovirals (ARVs) for the treatment of HIV infection has been detected by a range of federal and state law enforcement agencies. ${ }^{2-5}$ Recent reports have documented widespread street markets for ARVs in South Florida, where vulnerable indigent patients are targeted by pill brokers to trade their ARVs for money and/ or drugs. ${ }^{6-8}$ Little is known about illicit ARV demand; however, emtricitabine/tenofovir disoproxil fumarate (FTCTDF; Truvada ${ }^{\circledR}$ ), the only U.S. Food and Drug Administration (FDA)-approved medication for pre-exposure prophylaxis (PrEP), is among the most frequently diverted according to recent reports by both law enforcement (Surratt HL, Kurtz SP: A national perspective on the abuse and diversion of prescription drugs, article presented at Nova Southeastern University Faculty Symposium. Ft. Lauderdale, Florida, 2013) and patients. ${ }^{9}$

Following publication of the results of the iPrEx clinical trial of FTC-TDF use for PrEP by men who have sex with men (MSM) in $2010,{ }^{10}$ Centers for Disease Control and Prevention (CDC) interim guidance for PrEP for high-risk MSM in 2011, ${ }^{11}$ and FDA approval of FTC-TDF for PrEP in $2012,{ }^{12}$ CDC issued detailed PrEP clinical guidance for healthcare providers in 2014. ${ }^{13}$ Uptake of PrEP by MSM has been slow, which has been attributed to lack of knowledge of PrEP; healthcare provider concerns about frequent health monitoring and patient adherence; variable levels of coverage by governmental and private health insurance; and stigma within MSM communities. ${ }^{14,15}$ To date, no national public health campaign has addressed MSM with accurate information about the purpose, efficacy, accessibility, and adherence requirements of PrEP (although CDC recently announced partnerships with 12 state and local health departments that will include the targeted expansion of PrEP use among MSM) ${ }^{16}$ In addition, the manufacturer has thus far decided not to promote the use of FTC-TDF for PrEP. ${ }^{17}$ Communitybased organizations in some urban areas have worked to fill the information gap, but lack national reach.

In the absence of a rapid and broad-based public health response following the approval of FTC-TDF use for PrEP among MSM, there is concern that incomplete knowledge of PrEP use and health monitoring requirements, as well as the high cost of the medication, may lead vulnerable MSM to seek access to FTC-TDF through ARV street markets. ${ }^{6}$ In fact, the nonprescribed use of ARVs for prevention has been documented among high-risk MSM since at least 2004. ${ }^{18,19}$ In this study, we describe the findings of new qualitative research with highly vulnerable HIV-negative/unknown substance-using MSM in Miami, Florida, which yielded important data about men's understanding and use of PrEP, and potential linkages between ARV street markets and demand for PrEP.

ARSH: Center for Applied Research on Substance Use and Health Disparities, Nova Southeastern University, Ft. Lauderdale, Florida.

(C) Steven P. Kurtz and Mance E. Buttram, 2016; Published by Mary Ann Liebert, Inc. This Open Access article is distributed under the terms of the Creative Commons Attribution Noncommercial License (http://creativecommons.org/licenses/by-nc/4.0/) which permits any noncommercial use, distribution, and reproduction in any medium, provided the original author(s) and the source are credited. 


\section{Methods}

Data are drawn from focus groups conducted in 2014 to solicit information about the HIV testing and prevention practices of MSM at high risk for HIV. Men were recruited in Miami, Florida, through GPS-based social networking applications (GSNAs, e.g., Grindr, SCRUFF) and screened by telephone to determine if they met eligibility requirements: 18-35 years of age, HIV negative/unknown, had anal sex with two or more male partners in the past 90 days, reported drug use and/or binge drinking in the past month, and were current users of one or more GSNAs designed for MSM. Thirty-one men participated in six focus groups of three to eight participants each. Seventeen participants were Hispanic, seven African American/black, six white, and one other race/ethnicity; mean age was 27.2 years, which was similar across racial/ethnic groups. Focus group discussions lasting about 90 minutes were conducted in person in a research field office in Miami and audiorecorded for later transcription. Written informed consent was administered using a protocol approved by Nova Southeastern University's Institutional Review Board. The study was conducted in accordance with the Helsinki Declaration of 1975, as revised in 2008. The discussions were guided by a semistructured interview schedule that included open-ended questions about the men's knowledge of HIV disease, treatment, and transmission risk behaviors; HIV and sexually transmitted disease testing and prevention practices; and the linkages between substance use and sex. PrEP knowledge, access, and use emerged as a topic of discussion in the first focus group. Thereafter, when not spontaneously occurring, facilitators asked specifically about these questions, although PrEP was not the primary focus of inquiry. Participants were compensated $\$ 50$ for their participation and provided with basic information about the FDA-approved PrEP regimen.

Audiorecorded discussions were transcribed by an independent transcriptionist and reviewed for accuracy by the second author. Texts were entered into ATLAS.ti 7 software for coding and analysis. The analysis for this article focused on coding the themes and the texts of men's responses to questions about PrEP.

\section{Results}

Almost half of the participants had heard of PrEP or of the use of ARVs for pre-exposure prevention. Of those who had heard of PrEP, several men indicated a thorough understanding of PrEP as a daily regimen that was prescribed and monitored by a physician; one of these men indicated that he had requested and been denied PrEP by his doctor:

"I thought about taking [PrEP] as prevention, and I spoke to my doctor. He said he would only suggest it in extreme cases. They don't know what the long-term effects are of somebody negative taking the pill."

The majority of participants who had heard of it understood PrEP as a variation of a morning after pill, as described by the following three participants:

"You can have bareback sex all you want as long as you take these drugs. Right after you do it, though. It's like the plan B pill for us. Like girls, they got a plan B. Gay guys, we got the plan C."
"When that [news of] Truvada ${ }^{\circledR}$ got out, everybody is like girl, I can have sex without a condom. You know, give me the pill! You got a pill girl? Let me get a pill."

"You could fuck him without a condom and you can take the pill tomorrow."

Although several men knew friends who had taken PrEP informally, only one participant, who worked in the pornographic film industry, had personally done so:

\begin{abstract}
"This guy I filmed with, he was positive. He's undetectable on meds, and he had them all right there. He gave me two Truvada $^{\circledR}$, one for that moment, and one for the next day as a kind of preventative. I got tested two days afterward. I don't feel sick. I have a good idea I'm still negative, and I don't know if it's because of the Truvada ${ }^{\circledR}$, or because his viral load was low, or because I was topping him. That was the first time I was ever offered meds by a positive sex partner. I think that supporting each other like that is important. He's trying to help."
\end{abstract}

One participant indicated that some awareness among MSM of the potential efficacy of ARVs for prevention predates the completion of scientific studies, regulatory approvals, or public health announcements:

\begin{abstract}
"I had a friend, like maybe seven years ago, who actually did the same thing, who had sex with somebody who was HIV positive and thought that by taking their medication-the other person's medication-it would save them from being positive."
\end{abstract}

A number of participants were also familiar with street markets in ARVs:

R1: "It's [Truvada] very expensive, but most of these people who are HIV positive, they're undetectable, they're not taking their pills, they're selling it..."

R2: "... they sell it for their drug habit."

R1: "Who doesn't know somebody that's HIV positive, you know? 'Girl, sell me one of your pills! Or give me a couple of weeks' worth."

\section{Discussion}

The sample of men we enrolled was at high risk for HIV infection and would appear to meet criteria for highest priority for PrEP uptake. ${ }^{11}$ Nevertheless, few participants understood PrEP to be a medication prescribed and monitored by a physician, and the one participant who requested such a prescription was denied. Moreover, a majority of participants had never heard of PrEP at all.

Of even greater concern is the extent of misinformation about PrEP that we found. Among men who had heard about PrEP, most believed that it consisted of a drug to be taken before and/or immediately after sex and that it was generally acquired on the street or through HIV-positive friends. Although recent clinical trials have demonstrated the efficacy of an episodic PrEP regimen, ${ }^{20}$ there is continuing broad agreement among governmental agencies and scientists that individuals taking PrEP require frequent testing, regular health monitoring, and ongoing behavioral support. ${ }^{13,20}$

At the present time, important structural barriers exist for high-risk MSM to access these services, including the lack of 
accurate and accessible information and limited access to healthcare. ${ }^{6,21}$ Nevertheless, it is unsurprising that sexually active men like those in our sample are highly interested in PrEP, receive and transmit street folklore about PrEP, and are aware of PrEP access through informal routes. In addition to the lack of professional guidance and support for the efficacious use of PrEP, informal use would increase risk for inconsistent drug access, unmonitored restarts, use when unknowingly infected, and compromised or counterfeit pills in the supply chain. MSM are urgently in need of culturally relevant knowledge about the efficacious use of PrEP as well as clear avenues of access for the medication.

\section{Limitations}

Several limitations of the study design are important to note. Our study included a small convenience sample of men who met high-risk eligibility criteria. Both these aspects and the study location in Miami, Florida, limit generalizability of our results. For example, the State of Florida has high numbers of residents without health insurance and has not expanded Medicaid coverage, which may exacerbate the lack of information and uptake - and the extent of misinformation-about PrEP compared with other locations. Certainly, the collection of systematic data from larger samples across diverse geographic regions is needed to assess the scope and magnitude of ARV diversion and misuse, and of PrEP misinformation, more broadly. In addition, our data rely on self-report, and some respondents may have refrained from reporting socially undesirable behaviors in the context of a focus group discussion.

\section{Conclusion}

Even given these limitations, we believe that our findings of misinformation about PrEP among highly vulnerable MSM-together with research describing widespread street markets in $\mathrm{ARVs}^{7,8}$ - point to important public health policy concerns. First, the diversion and misuse-driven by drug cost-of approved medications to prevent epidemic lifethreatening infectious disease would appear to be unprecedented in developed countries. Second, the absence of a robust information campaign by either the manufacturer or national public health authorities that explains the efficacious use of FTC-TDF for HIV prevention may lead to nonprescribed unsupervised use of the medication that could reduce PrEP impact on the epidemic through the development of resistant virus and through unintentional transmission. National and community-based information campaigns and health navigation resources are critical to ensure that diverse at-risk groups are reached with culturally relevant knowledge. ${ }^{22}$ Third, strategies are needed to overcome other structural barriers to efficacious PrEP uptake, including the standardization of insurance coverage, and a reduction in cost disparities across the high-risk groups for whom PrEP could dramatically reduce the number of new infections.

Finally, should research confirm significant diversion and misuse of ARVs in diverse locations, the FDA should strongly consider requiring manufacturers of medications used for PrEP to (1) improve labeling to clearly address the risks of misuse, diversion, sharing, and trading of the medications and (2) conduct rigorous postmarketing surveillance to systematically monitor the diversion and misuse of the drugs.
Labeling and monitoring are common elements of FDA Risk Evaluation and Mitigation Strategies (REMS). Although the REMS for Truvada include a detailed patient medication guide, ${ }^{23}$ REMS for many other misused and diverted pharmaceutical products require postmarketing surveillance and label alerts to patients. ${ }^{24}$

\section{Author Disclosure Statement}

No competing financial interests exist.

\section{References}

1. Inciardi JA, Surratt HL, Kurtz SP, Burke JJ: The diversion of prescription drugs by health care workers in Cincinnati, Ohio. Subst Use Misuse 2006;41:255-264.

2. Dorschner J: Activists: HIV care scam uses homeless. Miami Herald 2005;30:1A.

3. Flaherty MP, Gaul GM: Florida Medicaid fraud costs millions, report says. The Washington Post 2003:E03.

4. Glasgow K: The new dealers: They're poor, black, and HIVpositive. Their product? The AIDS medications intended to cure them. Miami New Times 1999.

5. Kuehn BM: Reports: HIV drugs targeted for black market. JAMA 2014;312:1086-1087.

6. Kurtz SP, Buttram ME, Surratt HL: Vulnerable infected populations and street markets for ARVs: Potential implications for PrEP rollout in the US. AIDS Care 2014;26:411-415.

7. Surratt HL, Kurtz SP, Cicero TJ, et al.: Antiretroviral medication diversion among HIV-positive substance abusers in south Florida. Am J Public Health 2013;103:10261028.

8. Tsuyuki K, Surratt HL, Levi-Minzi MA, et al.: The demand for antiretroviral drugs in the illicit marketplace: Implications for HIV disease management among vulnerable populations. AIDS Behav 2015;19:857-868.

9. Kurtz SP, Surratt HL, Buttram ME: ARV diversion among HIV-positive MSM: Individual and market characteristics. Abstract 2039, presented at AIDS Impact Conference, Amsterdam. 2015. Available at www.aidsimpact.com/ 2015/Academics/Programme/abstract/?id=2039 Accessed November 24, 2015.

10. Grant RM, Lama JR, Anderson PL, et al.: Preexposure chemoprophylaxis for HIV prevention in men who have sex with men. N Engl J Med 2010;363:2587-2599.

11. Smith D, Grant RM, Weidle PJ, et al.: Interim guidance: Preexposure prophylaxis for the prevention of HIV infection in men who have sex with men. MMWR Morb Mortal Wkly Rep 2011;60:65-68.

12. Food and Drug Administration: Truvada for PrEP fact sheet: Ensuring safe and proper use. 2012. Available at www.fda .gov/downloads/newsevents/newsroom/factsheets/ucm312279 .pdf Accessed July 2, 2015.

13. Centers for Disease Control and Prevention: Preexposure prophylaxis for the prevention of HIV infection in the United States-2014: A Clinical Practice Guideline. 2014. Available at www.cdc.gov/hiv/pdf/prepguidelines2014.pdf Accessed April 13, 2015.

14. Liu A, Cohen S, Follansbee S, et al.: Early experiences implementing pre-exposure prophylaxis (PrEP) for HIV prevention in San Francisco. PLoS Med 2014;11:e1001613.

15. McNeil DG: Are we ready for HIV's sexual revolution? International New York Times 2014:8.

16. Centers for Disease Control and Prevention: Dear Colleague: September 25, 2015, Information from CDC's 
Division of HIV/AIDS Prevention. Available at www.cdc .gov/hiv/dhap/new/dcl/092515.html Accessed October 6, 2015.

17. Chen C: Gilead's pill can stop HIV. So why does almost nobody take it? Bloomberg Business. February 18, 2015. Available at www.bloomberg.com/news/articles/2015-0218/gilead-s-pill-can-stop-hiv-so-why-does-almost-nobodytake-it Accessed October 6, 2015.

18. Kellerman SE, Hutchinson AB, Begley EB, et al.: Knowledge and use of HIV pre-exposure prophylaxis among attendees of minority gay pride events, 2004. J Acquir Immune Defic Syndr 2006;43:376-377.

19. Mansergh G, Koblin BA, Colfax GN, et al.: Preefficacy use and sharing of antiretroviral medications to prevent sexuallytransmitted HIV infection among US men who have sex with men. J Acquir Immune Defic Syndr 2010;55:e14-e15.

20. Molina JM, Capitant C, Spire B, et al.: On demand PrEP with oral TDF-FTC in MSM: Results of the ANRS Ipergay trial. Abstract Number 23LB. Presented at the Conference on Retroviruses and Opportunistic Infections, Seattle, Washington, 2015. Available at: www.croiconference.org/ sessions/demand-prep-oral-tdf-ftc-msm-results-anrs-ipergaytrial Accessed October 6, 2015.

21. Curran JW, Crosby RA: Pre-exposure prophylaxis for HIV: Who will benefit and what are the challenges? Am J Prev Med 2013;44:S163-S166.
22. Mutchler MG, McDavitt B, Ghani MA, et al.: Getting PrEPared for HIV prevention navigation: Young black gay men talk about HIV prevention in the biomedical era. AIDS Patient Care STDS 2015;29:490-502.

23. Food and Drug Administration: Risk Evaluation and Mitigation Strategy for Truvada. Available at: www.fda.gov/downloads/ Drugs/DrugSafety/PostmarketDrugSafetyInformationfor PatientsandProviders/UCM312304.pdf Accessed October 6, 2014.

24. Food and Drug Administration: FDA announces safety labeling changes and postmarket study requirements for extendedrelease and long-acting opioid analgesics. September 10, 2013. Available at www.fda.gov/NewsEvents/Newsroom/ PressAnnouncements/ucm367726.htm Accessed October 6, 2015.

Address correspondence to: Steven P. Kurtz, PhD ARSH: Center for Applied Research on Substance Use and Health Disparities Nova Southeastern University $2 \mathrm{NE}$ 40th Street, Suite 404 Miami, FL 33137

E-mail: steven.kurtz@nova.edu 